\title{
Developing Contextual Social Awareness in Engineering: Placing human diversity and social justice at the Center of the Engineering Process
}

\section{Mrs. Greses Perez, Stanford University}

Greses Pérez-Jöhnk is an engineer, educator, and a Ph.D. candidate at Stanford University in engineering education, Science Education and Learning Sciences and Technology Design. Her research interests include the education of Black and Brown students, especially those from immigrant communities, in the fields of engineering and science. In addition to her ongoing work on culturally relevant VR science teaching, Greses seeks to understand issues of diversity and inclusion in engineering. Specifically, she investigates how language influences who engages in the technical fields. She was recently awarded the Stanford DARE fellowship. Globally, she is part of the Galapagos research-practice partnership that seeks to improve the teaching of science for underserved communities through education for sustainability. Before coming to Stanford, she was a bilingual educator at Plano ISD. In Plano, she served in the Gifted and Talented Advisory Committee and the Elementary Curriculum Design team. Prior to starting her career in education, Greses was a project manager for engineering programs funded by the European nonprofits in the Caribbean. She holds a B.S. in Civil Engineering from Santo Domingo Technological Institute, a M.Eng. in Environmental Engineering from the University of Puerto Rico at Mayagüez, and a M.Ed. in School Leadership from Southern Methodist University.

\section{Mr. Patrick Marcel Danner, Technical University of Munich (TUM)}

Patrick Danner is a graduate student in Chemistry M.Sc. and Technology \& Management M.Sc. at the Technical University of Munich and a Visiting Graduate Researcher at Stanford University. His research interests are at the intersection of science, technology transfer, biases and diversity in design and start-up context. His previous work before Stanford, includes research on novel 2D nano-materials, research in the field of catalysis as well as investment analyst at an early-stage venture capital fund.

\section{Dr. Shannon Katherine Gilmartin, Stanford University}

Shannon K. Gilmartin, Ph.D., is a Senior Research Scholar at the Stanford VMware Women's Leadership Innovation Lab and Adjunct Professor in Mechanical Engineering at Stanford University. She is also Managing Director of SKG Analysis, a research consulting firm. Her expertise and interests focus on education and workforce development in engineering and science fields. Previous and current clients include the American Chemical Society, the Anita Borg Institute for Women and Technology, California Institute of Technology, the College of Natural Sciences and Mathematics at California State University Fullerton, the Office of the Vice Provost for Graduate Education at Stanford University, the School of Medicine at Stanford University, and the School of Fisheries and Ocean Sciences at the University of Alaska, Fairbanks.

\section{Dr. Carol B. Muller, Stanford University}

Carol B. Muller is the Executive Director of WISE Ventures, an internal initiative at Stanford located in the Office of Faculty Development, designed to communicate, build networks, and help amplify existing and seed new and needed ventures across the Stanford campus to advance equity in science and engineering. She also serves as executive director for Stanford's Faculty Women's Forum. A longtime university administrator, educator, and social entrepreneur, she served as Associate Dean for Thayer School of Engineering at Dartmouth, where she co-founded the campus-wide Women in Science Project in 1990. She founded and served as chief executive of MentorNet, a large-scale online nonprofit global mentoring network advancing diversity in engineering and science (1996-2008). At Stanford, she was consulting associate professor of mechanical engineering between 1998 and 2002, collaborating with faculty and staff to create "New Century Scholars: Teaching, Learning, and Your Academic Career," a summer workshop designed for new engineering faculty members. A Fellow of the Association for Women in Science, Dr. Muller and her work have been recognized with other national awards, including the Presidential Award for Excellence in Science, Mathematics and Engineering Mentoring, and the Anita Borg Social Impact 
Award. She has authored and presented numerous papers, presentations, and workshops. She earned a bachelors degree from Dartmouth (majoring in philosophy), and masters and Ph.D. degrees in education administration and policy analysis at Stanford, and continues to build upon research in the design and implementation of programs.

\section{Dr. Sheri Sheppard, Stanford University}

Sheri D. Sheppard, Ph.D., P.E., is professor of Mechanical Engineering at Stanford University. Besides teaching both undergraduate and graduate design and education related classes at Stanford University, she conducts research on engineering education and work-practices, and applied finite element analysis. From 1999-2008 she served as a Senior Scholar at the Carnegie Foundation for the Advancement of Teaching, leading the Foundation's engineering study (as reported in Educating Engineers: Designing for the Future of the Field). In addition, in 2011 Dr. Sheppard was named as co-PI of a national NSF innovation center (Epicenter), and leads an NSF program at Stanford on summer research experiences for high school teachers. Her industry experiences includes engineering positions at Detroit's "Big Three:" Ford Motor Company, General Motors Corporation, and Chrysler Corporation.

At Stanford she has served a chair of the faculty senate, and recently served as Associate Vice Provost for Graduate Education. 


\title{
Developing Contextual Social Awareness in Engineering: Placing human diversity and social justice at the Center of the Engineering Process
}

\begin{abstract}
Can a deeper understanding of human diversity improve engineering processes and outcomes? If so, can we teach these social dimensions within the timeframe of a course? A predominant focus on technical aspects in the teaching and learning of engineering coupled with cultural stereotypes of who can become an engineer leave many at the margins of the field rather than at the center of it. This research paper investigates how participation in the ENGR-Diversity course may build students' awareness of social aspects in engineering. Through an examination of students' design considerations, we provide empirical data to two research questions (1) To what extent do students consider social issues in design problems? (2) What are the changes, if any, in students' social awareness after they participate in a course experience on culture, diversity, and equity in engineering communities?
\end{abstract}

Participants included 74 students at a university in California enrolled in the course during the 2019 winter quarter. Through a mixed-methods approach, we investigated how culture and diversity shape engineering work. Data sources included students' responses to the Palo Alto Flooding Problem, an exercise inspired by the well-known Midwest Flooding Problem by Atman and colleagues. The problem was administered at the beginning (pretest) and end of the course (posttest).

The research team undertook analyses of overall responses, segments, and Contextual Social Awareness (CSA) segments. Through a two-tailed paired t-test of students' responses and a twoway analysis of variance of groups' change on CSA segments, we examine the extent to which students considered social issues in their responses and their changes in social awareness. Our findings suggest that engineers will consider the lived experiences of people and their communities in their work when they become aware of the role of broader societal issues in engineering, thereby opening up possibilities for more equitable solutions. Reported results suggest that students can change their design considerations about social issues in engineering within a short time frame.

The proposed binary Contextual Social Awareness (CSA) expands the scope and depth of the classic MWF framework. The CSA adds valuable insights for researchers and educators into the nature of responses. The most interesting insights of this work resulted from centering the user's voices by closely investigating the original quotes from students. This study is a further example that it is always worthwhile to invest time to deeply understand the participants, especially when it comes to engineering design.

Keywords: Contextual Social Awareness, engineering design, diversity, social justice, engineering education 


\section{Introduction}

Can a deeper understanding of human diversity improve engineering processes and outcomes? This research investigates an engineering course focused on culture, diversity, and equity and how it may build contextual social awareness in students. Through an exploration of students' responses to design problem scoping, the study considers 1) if and how students incorporate aspects of human diversity in engineering work and 2) whether awareness of such social aspects can be learned within a short timeframe.

In this work, we focus on human diversity as reflective of "broad heterogeneity in social identities and statuses represented among individuals in a shared engineering experience" [1]. We see these dimensions as situated in, interacting with, and influenced by the cultural and social norms in which individuals operate. In turn, individuals affect those cultural norms. Understanding these aspects is increasingly recognized as an important part of learning to become an engineer. Though traditional engineering education has been, and to a large extent still is, focused on students acquiring technical knowledge [2] [3], in the workplace engineers are required to bring more than technical expertise to solve problems. As part of their work, they often draw on different disciplinary content, kinds of skills, and knowledge that is contextual, social, tactical, and explicit [2]. Engineering work is in fact a highly social activity despite cultural currents that emphasize technical vs. social elements [4] [5].

Focusing on dimensions of diversity in engineering education matters because of the central role of understanding stakeholders in solving problems, the dynamic nature of contexts, and issues of representation in the field. First, current design frameworks have shown the value of placing users at the center of solving engineering problems [6]. In following most design approaches, understanding users and stakeholders involved is one of the first tasks engineers undertake in creating a new product. More specifically, engineers need to consider the wide range of needs and wants of those involved, which may not all be the same. Second, the dynamic nature of conditions surrounding and integrated with most engineering problems demands adaptability from engineers. The technological, economic, social, regulatory and political changes create what engineering scholars have referred to as the VUCA (Volatile, Uncertain, Complex and Ambiguous) world [7]. VUCA results from rapid changes in what people want as well as in the sociopolitical landscape of societies. Under such circumstances, engineers need to adapt continuously to changing needs by remaining in contact with project stakeholders and assessing relevant changing conditions. For engineers, assessing relevant changing conditions requires them to understand the dimensions of diversity in engineering, often requiring them to stay in more frequent contact with those for whom they are designing (e.g. client, end users, and those impacted by the solutions) [8]. According to a recent CBInsights Report, such changes are already impacting industry [9]; the report identified empathy in design as one of the 14 most important trends for technology companies in 2020.

The third reason pertains to representation in the engineering field. The low representation of women and members of several racial and ethnic minority groups in engineering in the United States is well-documented [10], [11]. This mismatch in diversity between engineers and the general U.S. population also supports the need to educate engineers about the role of social aspects in their work. The United States has significantly fewer women 
and people of color (e.g., African-American, Native American, Latinx, and other racial and ethnic backgrounds) among engineering degree earners relative to the national picture [11]. Cultural beliefs and stereotypes about who can be an engineer, who belongs in engineering, and who will succeed in these spaces prove to be a major obstacle to enabling engineering to catch up with changing demographics in the population and workforce [4], [10].

We argue that increasing awareness among engineering students (and students more generally) of the roles of human diversity in their work has the potential for (at least) two positive outcomes: increasing the diversity of ideas at the "engineering table" (by expanding the range of lived experiences of engineers at the table), and increasing the access to an engineering pathway of more diverse students [12], [13]. Of course these two outcomes are intrinsically interconnected. Both also have the possibility of moving us towards a society that is more socially just. If engineers effectively incorporate diversity into their work, there is the very real possibility of bringing social justice considerations to the processes and outcomes of engineering. In this context, social justice is the fair and just relation between individuals and society when considering differences, power, and privilege [14]. We are better equipped to address social injustices when we disrupt systems designed to oppress, dominate, and dehumanize individuals and communities on the basis of their diversity [15].

The leap from diversity to social justice is not immediate. In order for this connection to be made, "difference", or more specifically, the way people construct "difference", must be seen as both a source of creativity and a lever of major inequality and injustice in the world. Only when diversity is discussed alongside power and privilege can those injustices be addressed [14]. Educating engineers about diversity and social justice issues in their work means preparing them to look inward to the profession and critically assess how engineering practices reflect bias and protect those in power. Developing this understanding means giving engineers the tools to ask: who benefits from which types of solutions designed by whom--and is this just? Not having a large part of society participate in engineering problem-solving, particularly given the different social experiences of diverse groups, results in missed opportunities and inefficient or ineffective product design [12]; the importance of diversity and the resulting competitive advantage for companies through diverse teams is widely discussed and recognized in the literature [16]. But there is a social justice cost on a vast scale too, as "missed opportunities" can mean continuation of poverty, hunger, oppression, and environmental decimation.

In this research, we look at aspects of diversity in students' work and whether such social aspects can be learned within the timeframe of a course. A predominant focus on technical aspects in teaching and learning engineering [2], [17], combined with cultural stereotypes of who can become an engineer, leave many at the margins of solutions rather than at the center of them [18], [19]. Less attention has been given to incorporating into the curriculum the myriad of societal factors that influence engineering processes and products. Our research suggests that engineers will consider the lived experiences of people and their communities in their work when they become aware of the role of broader societal issues in engineering, thereby opening up possibilities for better and more equitable solutions. We examine the case of a single course designed to connect engineering practice to questions of culture and diversity. In addition, we also consider how students' learnings in this course potentially support the development of more "socially aware" design. 


\section{Background of the Study}

As the engineering profession is permanently changing, engineering education constantly needs to adapt to the changes in engineering work. Current shifts from a focus purely on technical aspects to more contextual and social aspects pose a challenge both in engineering education and research. Practitioners and scholars grapple with questions around the applicability of this new paradigm of developing greater social awareness in engineering as a means to improve engineering solutions. For instance, one may ask how can the effectiveness of factors such as social understanding of design context be accurately measured? And if there are no accurate measures, how can educators understand the potential impact of courses on students' performance as engineers? Previous researchers attempted to measure social awareness of mechanical engineering students by analyzing responses to questionnaires before and after a course experience [20]. Others used questionnaires to measure self-awareness and social awareness of engineering students in human-centered design [21]. For instance, Cech [22] developed a questionnaire where engineering students self-reported their perceived importance of different social aspects within their work. According to the author, social welfare importance decreases over the years in the engineering program. Cech argued for new educational programs with the goal of highlighting the social impact of engineers and increasing their social awareness.

Instead of relying on questionnaires and self-reported Likert-scale questions, Atman [23] developed a framework to assess design problem scoping capabilities of engineers based on a general design test, known as the Midwest Flooding (MWF) problem. The goal of the task is to understand which factors are considered when working on design problem scoping and whether these factors are related to context or detail. Atman's work showed that, among first-year students, self-identified women tend to consider contextual factors more than do men. Seniors provided a greater number of answers, of all types, than first-year students [23]. Other methods to measure design capabilities are tasks to design a structure to launch a Ping-Pong ball to hit a target with specific dimensions or a task to design a crossway for a busy street on campus [24], [25]. These tasks differ in specificity and freedom to design. As the least structured of these tasks, the MWF requires students to think more broadly about the task [26]. Designing a playground is another example of a less structured design task, similar to the MWF.

Research considering all these design tasks shows that those with more experience engage in higher quality design processes, in terms of the type, quantity and nature of factors considered in the design. They also show that good design processes require both technical and detail focused skills but also the ability to consider complex aspects. In first-year students, the context thinking skills are mostly less developed compared to seniors or professionals [23], [24], [25]. While the MWF problem provides opportunities to assess an overall design focus, it might not go far enough in assessing skills more recently recognized as critical to effective engineering processes and results, such as awareness of different cultural backgrounds, power structures, local users, socioeconomic status, access to resources for different groups of people, diversity (e.g. perspectives in and out engineering, biases about people and communities) and the inclusion of underrepresented minorities in the engineering field. These aspects of social awareness become central in current design problems. However, it is difficult to assess students' learning about the importance of social aspects in design [20], [21], [22] in new ways that are not based just on self-reported questionnaires, but also on task bases. Such scholarship could open 
the door for the engineering education community to teach about social awareness more effectively. This paper presents an approach for measuring social awareness based on the extended MWF task.

\section{Research Questions}

This study investigates the characteristics of more socially aware designs. To explore this theme, we seek to generate and analyze empirical data to answer the question: What does social awareness "look" like before and after a course designed to bring topics of diversity, culture and inclusion in engineering design front and center? Our study design does not seek to establish a causal relation between social awareness and the course. Instead, it offers an initial exploration of potential elements in "socially aware design". We examine changes in students' understanding of social aspects in engineering work as a result of a course experience. We pose two research questions:

- To what extent do students consider social issues in design problems?

- What are the changes, if any, in students' social awareness after they participate in a course on culture, diversity, and equity in engineering communities?

\section{Methods}

\section{Overview of the course and research design}

Our study took place in the context of a 10-week long engineering course at a research university in the 2019 winter academic term. We refer to the course as ENGR-Diversity for the purpose of this paper, a course that had been offered since 2015, and was taught in 2019 for the fourth time. Eighty-two students participated in the 2019 offering of the course, but we only report data from 74 of its students who participated in all research and evaluation data collection activities (described below).

The course touched on a number of, but by no means all, dimensions of human diversity. Through guest speakers, readings, discussions, and a final project, the course highlights the interplay of diversity with power and privilege in engineering. As described in the University Course Catalogue, some of the considerations embedded in the course related to human diversity include: "how cultural beliefs about race, ethnicity, gender, sexuality, abilities, socioeconomic status, and other intersectional aspects of identity interact with beliefs about engineering, influence diversity in the field, and affect equity in engineering education and practice." Additionally, students in ENGR-Diversity explore how engineering cultures and environments respond to and change with individual and institutional agency.

In our research, we asked the students in ENGR-Diversity to complete a modified version of the Midwest Flooding Problem (MWF), renamed the Palo Alto Flooding Problem (PAFP) [27]. Participants completed the PAFP one time in the first week of class (pretest) and a second time before the last week of class, week 9 (posttest). The task was presented to students as follows: "Over the winter, the cities of East Palo Alto and Palo Alto experienced massive flooding of the San Francisquito Creek. What factors would you take into account in designing a 
retaining wall system for the San Francisquito?" The task was changed slightly from the original MWF to set it into a local context with social equality discrepancies between the two cities.

The PAFP pretest was sent and completed online by students during the first week of the course at the time of their choosing within a 24-hour turnaround period. For the pretest, students had up to ten minutes to complete the task but could stop anytime. In the task, students list factors they would want to account for in the design problem scoping. After ten minutes, the test was automatically stopped. Students' demographic data were collected on the pretest. The posttest was performed in the same way as the pretest but during one of the last sessions of the course, in the ninth week of the quarter. Additional evaluation questions about the course were included on the posttest. Our method differs from the MWF problem, in which participants' written answers were supplemented with their think-aloud responses. Additionally, in our case, the design problem was presented in a Qualtrics survey form with a chronometer function to ensure standardized timing and an image of the San Francisquito creek and the Palo Alto communities.

Students' pre- and post-responses were analyzed following protocols developed by Atman and colleagues [23]. Two different authors coded the responses and achieved an interrater agreement of over 80 percent for both tests. Within this framework, each factor mentioned by students as they responded in writing to the problem is coded along the physical location (wall, water, bank, surroundings) and the frame of reference (technical, logistical, natural, social). Figure 1 shows an example of pre- and post-responses coded for physical location and frame of reference for one of the students. Therefore, a code-pair (physical location, frame of reference) represents each factor mentioned by students as they responded to the design task.

Segments are the number of different factors given by students when responding to the written task. Segments are split into detailed segments and context segments. The code-pair determines if the segment is a detail segment or a context segment. All code-pairs with combinations of wall or logistical, as well as water $\&$ technical are detailed segments, while all others are context segments [23].

A node represents a code-pair. Nodes reflect how many different code-pairs students considered in the exercise. The nodes coverage range from 0 to 24 . There are two types of nodes: detail nodes and context nodes. For example, we coded arguments around the material used for the wall as "wall, technical". If one time material is mentioned, it is a segment and a node. If a second time, material is mentioned with a new perspective (not just a repetition), we again coded it as "wall, technical". The student has now two segments but still one node, as they are the same code-pair. Both of these segments are detail segments and the code-pair is a detail node.

To deepen the investigation, and as a result of seeing post-responses that did not quite fit into the standard Atman coding protocol as described above, we expanded the original protocol to include the binary code Contextual Social Awareness (CSA). The team coded students' responses with one or zero. If the participants considered humans and their communities, their responses were coded with one. Otherwise, the responses were coded with zero if the ideas were not related to social aspects. Every segment is given a zero or one depending on whether or not participants incorporated societal aspects in their responses. In theory, a student could have a 
Contextual Social Awareness score equal to the number of segments they contributed in the design. The next section describes in detail the expansion of Atman's framework.

\section{The need for another measure: Contextual Social Awareness (CSA)}

During the coding, we noticed that the coding framework of the Midwest Flooding Problem [23] did not fully capture the changes in students' responses. For instance, on a first analysis pass Jose's ${ }^{1}$ answers appear to be relatively similar in the pretest and posttest according to the classic MWF framework (see Figure 1). His responses represent typical results for students in our class. Using the classical MWF framework, we do not see any significant differences, which suggest that Jose's ideas about the design task are constant in the pretest and posttest.

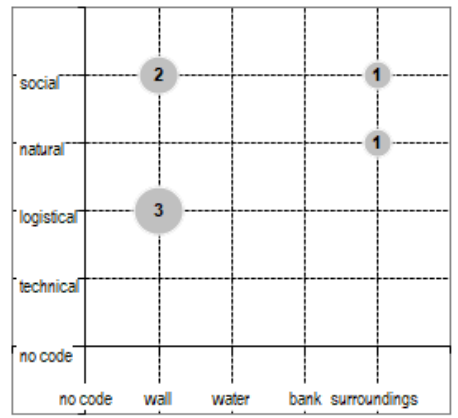

(a)

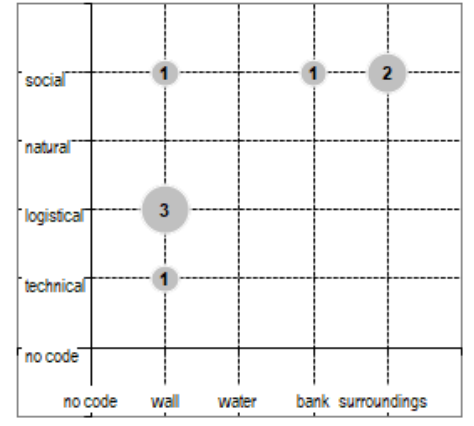

(b)

Figure 1. Pretest (a) and posttest (b) of Jose.

However, looking at Jose's responses more deeply against both the MWF coding framework and the goals of the ENGR-Diversity course, some parallels can be identified. In the pretest, three of Jose's answers focused on the effectiveness and cost of the wall (Wall, Logistical) and two responses in the walls' aesthetic characteristics (Wall, Social). In contrast, in the posttest, his answers incorporated logistical aspects as well as notions of power and influence. For instance, in the posttest, instead of framing the budget as restrictions, he provided an answer coded as (Wall and Logistical) where he focused on sources of funding. He noted, "Who would be funding this?" He also considered who is participating in the design process, he stated, "Who [is] represented on the team that is making the decision?" This segment was also coded as (Wall and Logistical). Furthermore, in his post-response, he also considered the community and the implications of the design work. He wrote, "What is the status of gentrification in the area?", which was coded as (Surroundings and Social). Jose raised issues of participation, community and power structure in his posttest - demonstrating, in other words, a deeper understanding of contextual and social issues. These changes are not fully captured in the initial Midwest Flooding Problem coding scheme.

\footnotetext{
${ }^{1}$ All names are pseudonyms, gender and pronouns are randomized.
} 


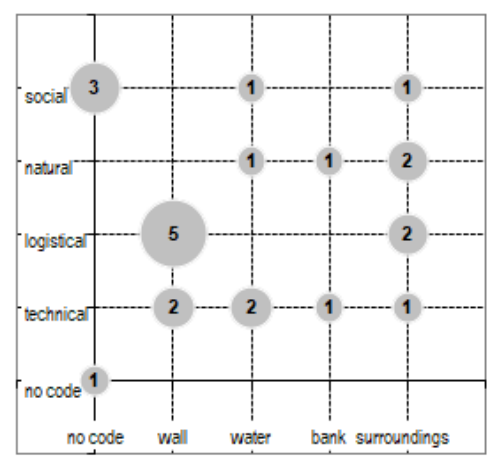

(a)

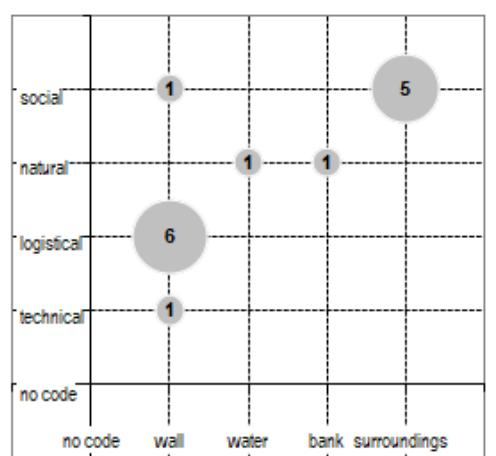

(b)

Figure 2. Pretest (a) and posttest (b) of Yulin.

The quotes from Yulin are another example for the need of an additional measure (see Figure 2). In the pretest, Yulin asked about the natural weather and technical factors. Some of these factors included references to the "historical and projected rainfall patterns" (Water, Natural), "potential impacts to the local ecology" (Surroundings, Natural) and "Seismic loads" (Bank, Technical). In the posttest Yulin still considered natural aspects; she wrote "[...] including geographical (e.g. landscape, seismic activity)", which we coded as (Bank and Natural). However, similar to Jose, she showed a higher number of CSA segments as she reflected about the socioeconomic status of the community. She stated: "Can the people we chose to displace afford to handle something like this" (Surroundings, Social). When referring to the design process, she said, engineers need to "pay particular attention to capturing traditionally marginalized voices" (Surroundings, Social). As in the example of Jose, there is an increased social awareness linked to socioeconomic status and minorities, which are issues at the heart of the ENGR-Diversity course. Jose's and Yulin's increase in Contextual Social Awareness (CSA) segments were not isolated cases but a trend in most students' responses.

Overall, posttest responses from students, in the course, included topics that had not previously been captured by the MWF exercise. Answers from participants reflected core ideas of the ENGR-Diversity class. Some of the topics reflected in students' answers include ${ }^{2}: 1$ ) awareness of local users, both understanding the impact, needs and wants of those who will benefit, be involved in, or be impacted by the design, 2) users socioeconomic status, 3) culture of users, engineering, and community, 4) issues of power structures and representation, or traditionally minoritized communities voices being heard at the engineering table, 5) access, or opportunities for individuals and communities to participate in the engineering enterprise, 6) diversity, or the broad heterogeneity in social identities and statuses of individuals and communities, 7) minorities, or groups traditionally underrepresented in engineering, and 8) separation of communities, the segregation of groups based on diversity aspects and/or issues of gentrification. Respondents mentioned one or more of these topics, which contributed to the construct of the new CSA variable. Although these topics were not used as codes, they made clear for us the need for a new measure to understand students' changes after the course. Even though the code "social" exists in the MWF exercise, it does not capture these types of topics adequately. In the MWF, the term "social" refers to aesthetics, safety of people, travel, damage

\footnotetext{
2 These themes would be elaborated in a future manuscript.
} 
to property, tourism, floodplains concerning people, infrastructure or agriculture. In contrast, some of the topics we noticed in students' responses show an awareness of issues deeply rooted in society around topics of diversity and social justice. Thoughts about these social justice issues demonstrate a broader awareness that goes beyond the context of the flooding prevention project.

To grasp better the difference in the framework proposed here, it is helpful to go back to Jose's and Yulin's original sentences. Following the MWF framework, we coded the segment "who [is] represented on the team that is making the decision?" as (Wall and Logistical). This answer was coded as Wall and Logistical because it discusses the team and a wall related project management topic [23]. However, it is not simply a project management idea as it also reflects Jose's thoughts about two of the identified eight social themes, namely: diversity and representation. It is important to point out that this quote would not be coded as Wall and Social in the MWF as this code-pair only covers issues around the wall aesthetics, the effects of the wall on people's ability to travel and/or the safety of the people. Yet, the code-pair Wall and Logistical is a reduction of Jose's statement that does not reflect his awareness for a broader context. This becomes at least a problematic reduction as quotes such as: "and gathering sufficient manpower ${ }^{3}$ to get the job done" are also coded as Wall and Logistical. Therefore, both sentences are treated the same way in the MWF framework, even though Jose's response showed a remarkable and important further social aspect.

In Yulin's response "can the people we chose to displace afford to handle something like this", coded as Surroundings and Social, she demonstrated concerns about displacements of people due to the project [23]. She takes this concern further by raising the question of socioeconomic status, which is one of the eight identified social themes. The question "Would it displace too many people?" is also coded as Surroundings and Social. This last response does not show further consideration of the identities of the displaced people, but rather suggests a view that sees the community as homogenous. As both sentences are coded identically in the MWF, this case too neglects the important aspects of social justice.

As these eight themes all capture social issues and demonstrate a broader contextual awareness, it was necessary to expand the MWF framework to include these important nuances. Here, we added a binary variable called Contextual Social Awareness (CSA) to the classic MWF framework, to represent all these social justice topics in one binary variable. This binary variable allows us to gain a deeper insight in the statistical as well as the visual representation (see Figure 3), compared to the standard MWF (see Figures $1 \& 2$ ). Students received a value of " 1 " on this new CSA measure for every answer that reflected social aspects and human diversity, either in their pretest or posttest, and " 0 " for any other type of answer. For example, on the pretest, Jose received a total CSA score of " 0 ", whereas on the posttest, he received a total CSA score of "6", given that six of his post-test responses incorporated contextual social awareness aspects.

In summary, the MWF provides a coding framework that allows segments to be labeled as code-pairs (e.g., logistical-wall, social-surroundings). These code-pairs are classified as context or detail. Yet, the well-established MWF coding does not account for social-justice oriented segments. The new CSA coding described above involves reviewing segments to see if they reflect the eight social justice themes listed above. This is a coding process that is done

\footnotetext{
${ }^{3}$ We did not code for gender-biased language even within CSA scores of 1.
} 
independently of MWF coding, where one would determine what proportion of all segments are CSA (see Results section for more details). We noted that CSA interpretation can also be done in partnership with MWF coding to see how the CSA label, in conjunction with code-pair labeling, makes a particular segment fall into the Context or Detail category as shown in Figure 3.

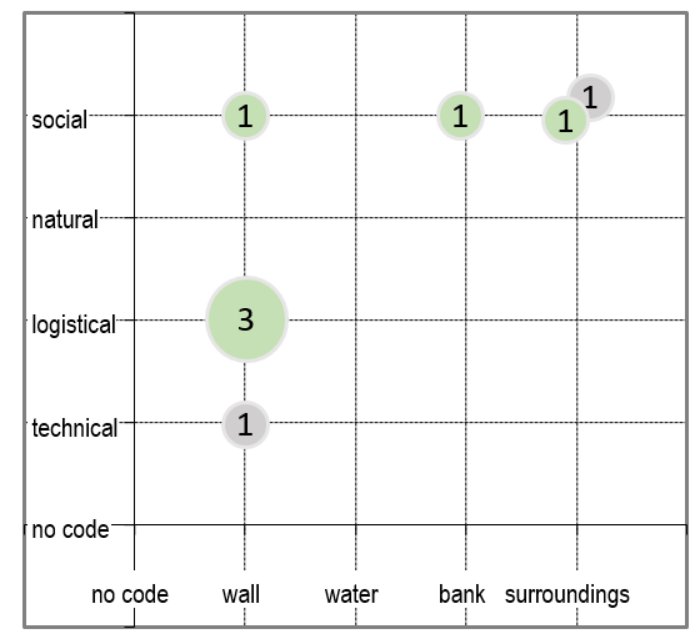

CSA answer

non-CSA answer

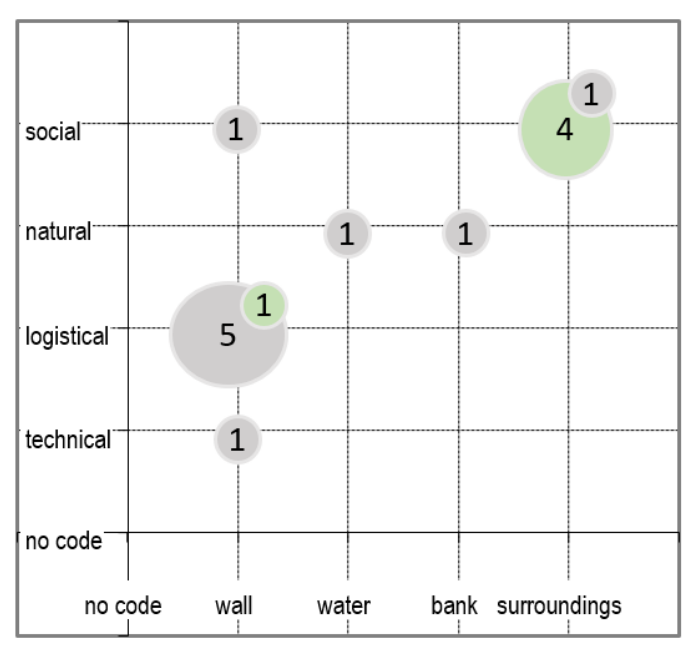

(b)

Figure 3. Posttest of Jose (a) \& Yulin (b) with contextual social awareness variable.

\section{Participants}

All 82 students who enrolled in the course "ENGR-Diversity" completed the pretest but only 74 of them answered the PAFP during week nine of the course (posttest). Only paired responses are reported in this manuscript. The pretest serves as our baseline and the initial analysis of those data are discussed in previous literature [27]. Table 1 shows the demographic information from students. ENGR-Diversity is an elective course, so it may have attracted participants who were predisposed to interests in issues of culture and diversity in the engineering profession. Some students may have taken the course perhaps to fulfill requirements rather than because they were interested. At the university where the course took place, computer science is considered an engineering degree. Only for the purpose of this study, we are creating a subgroup for computer science and another for engineering. 
Table 1. Demographics of participants in percentages*

\begin{tabular}{|c|c|c|c|c|c|c|c|}
\hline & \multirow[b]{2}{*}{ Categories } & \multicolumn{6}{|c|}{ Cohort } \\
\hline & & $\begin{array}{l}\text { 1st year } \\
\text { student }\end{array}$ & Sophomore & Junior & Senior & Graduate & Other \\
\hline Groups & Totals & $8(6)$ & $15(11)$ & $15(11)$ & $51(38)$ & $7(5)$ & $4(3)$ \\
\hline \multirow{2}{*}{ Gender } & Women & $5(4)$ & $5(4)$ & $8(6)$ & $27(20)$ & $3(2)$ & $4(3)$ \\
\hline & Men & $3(2)$ & $10(7)$ & $7(5)$ & $24(18)$ & $4(3)$ & - \\
\hline \multirow{6}{*}{$\begin{array}{l}\text { Race / } \\
\text { Ethnicity }\end{array}$} & Black & - & $4(3)$ & - & $3(2)$ & - & - \\
\hline & Latino(a) & $3(2)$ & $1(1)$ & $3(2)$ & $3(2)$ & $1(1)$ & - \\
\hline & Asian & $1(1)$ & $5(4)$ & $3(2)$ & $24(18)$ & - & $1(1)$ \\
\hline & White & $3(2)$ & $3(2)$ & $5(4)$ & $12(9)$ & $4(3)$ & $3(2)$ \\
\hline & Other & - & - & $1(1)$ & $3(2)$ & - & - \\
\hline & Multi-race/ethnicity & $1(1)$ & $1(1)$ & $3(2)$ & $7(5)$ & $1(1)$ & - \\
\hline \multirow{2}{*}{ URM } & URM students & $4(3)$ & $7(5)$ & $5(4)$ & $12(9)$ & $3(2)$ & $1(1)$ \\
\hline & Non URM students & $4(3)$ & $8(6)$ & $10(7)$ & $39(29)$ & $4(3)$ & $3(2)$ \\
\hline \multirow{4}{*}{ Major } & Engineering & $3(2)$ & $10(7)$ & $10(7)$ & $27(20)$ & $4(3)$ & \\
\hline & $\begin{array}{l}\text { Humanities / } \\
\text { Social Sciences }\end{array}$ & - & $1(1)$ & $1(1)$ & $3(2)$ & $3(2)$ & \\
\hline & Computer Science & $3(2)$ & $1(1)$ & $4(3)$ & $22(16)$ & - & \\
\hline & Undeclared & $3(2)$ & $3(2)$ & - & - & - & $4(3)$ \\
\hline
\end{tabular}

* The Total number of participants per category is in parentheses while the demographics are shown in percentages. Numbers may not add up due to rounding.

- Multi-race/ethnicity = More than one race or ethnicity with at least one of them URM. This category is a subset of the URM group. URM refers to underrepresented racial and/or ethnic minorities, such as African American, Latino/a/x, Native American, Pacific Islander and those who identify as multi-race/ethnicity.

- The cohort category "Other" indicates students who mark N/A to the cohort question.

\section{Analyses}

The research team undertook three different analyses: (i) analysis of overall responses, (ii) segments analysis, and (iii) Contextual Social Awareness (CSA) analysis. The overall responses and segments analyses help us understand the extent to which students consider social issues in design problems. The CSA analysis provides empirical data to the question of what are the changes in students' social awareness after their participation in the course. The first analysis looks at the pretest and posttest changes using a two-tailed paired t-test in the sample of students' responses $(n=74)$. We present the average answers by type and test as well as the average 
number of segments by node pair in this section. Using a two-sided dependent t-test, the second analysis determines whether the mean difference between paired observations on segments is statistically significantly different from zero. The paired two-sided t-test assumes that the groups perform differently in the two tests. In our case, we chose a two-tailed test because we wanted to investigate for the possibility of the differences being in both directions (increasing or decreasing). The third analysis expands on the previous one by comparing the means of Contextual Social Awareness from the same subgroups through a two-sided paired t-test. In addition, we also perform a two-way analysis of variance (ANOVA) of groups' change on CSA segments. These analyses do not seek to establish causal claims, instead it looks at change over time for our sample of ENGR-Diversity students.

\section{Results}

As in the analysis, the results section has three components: overview of overall responses, overview of segments, and CSA results. Table 2 provides an overview of students' answers and shows the average number of segments and nodes by type. Figure 4 also shows the average number of code-pairs for pretest and posttest. The student t-test results suggest no statistically significant differences between pretest and posttest, except for the context segments. The average context segments increased by 17 percent $(\mathrm{p}<0.05)$ with the code-pair social and surroundings as the main contributor of this increment (See Figure 4). This was the first indication that the overall sample increased their consideration to social aspects within the time frame between the two tests. However, we needed to understand the ways those considerations differ by groups.

Table 2. Average answers for pre- and posttest by segments and nodes types for overall sample

\begin{tabular}{cccc|ccc}
\hline & \multicolumn{3}{c|}{ Segments } & \multicolumn{3}{c}{ Nodes } \\
\cline { 2 - 7 } Instrument & Overall & Detail & Context & Overall & Detail & Context \\
\hline Pretest & 7.9 & 3.3 & 4.6 & 4.7 & 1.5 & 3.2 \\
\hline Posttest & 8.7 & 3.3 & 5.4 & 4.7 & 1.4 & 3.3 \\
\hline
\end{tabular}

Numbers might not add-up with the numbers form Figure 4 due to rounding. 

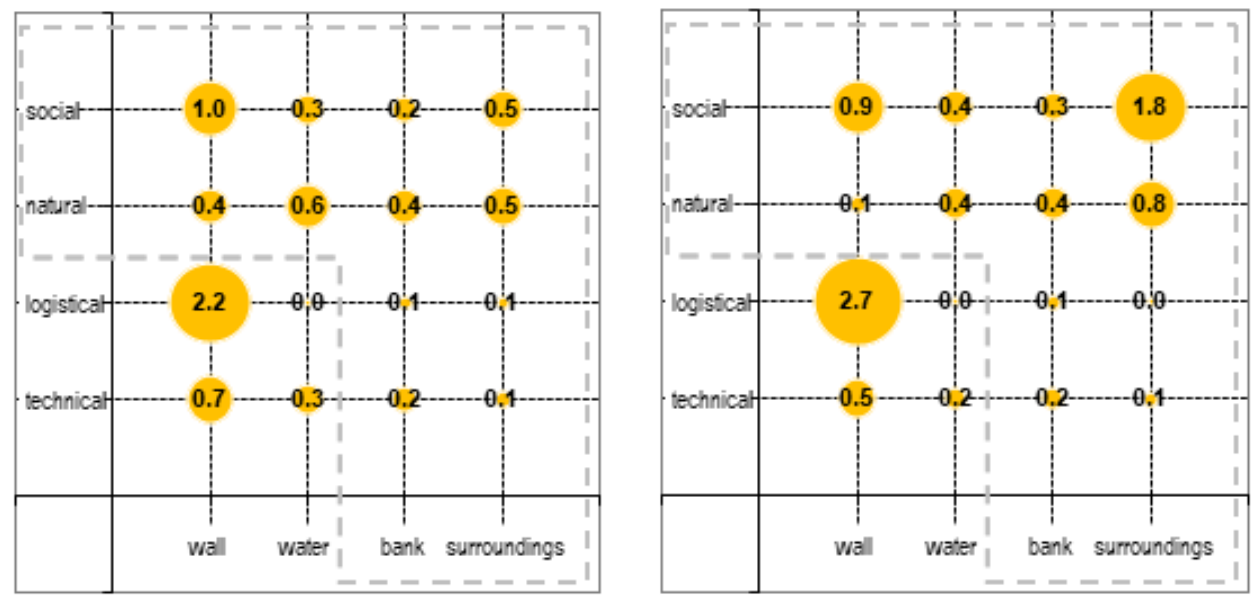

Figure 4. From left to right: Pretest and posttest average number of segments by code-pair for 74 participants in across-subjects comparison.

\section{Segments analysis}

The segment analysis compares the means from the observations at the beginning and end of the course from four different groups of participants clustered by gender, race/ethnicity, major and cohort. Through a two-sided paired t-test, this section provides answers to the question: Is there a difference in the segments outcome following a course experience?

Table 3 shows the absolute differences in the number of segments by type between pretest and posttest. Three different metrics are reported in the table: the average differences in number of segments, context segments, and detail segments. Each column represents the differences (posttest - pretest) for each metric (segments, detail and context) per student average for each of the identified subgroups (e.g. self-identified female). A two-tailed paired t-test was performed in these differences to determine if the means between paired observations differ from zero. The last column indicates the number of participants in each subgroup. We did not include in this paper supplemental analysis that suggests no statistically significantly changes between groups for the outcome segments. Therefore, we decided to focus our analysis on the changes within groups.

As shown in the table, we did not find significant differences in segments between observations based on gender. There is strong evidence $(p<0.05)$ that underrepresented minority $(\mathrm{URM})^{4}$ students increased the number of context segments. For this group, the number of context segments increased, on average, by approximately two segments. In supplemental analysis, not reported in this manuscript, we found that students who identified as Black showed the highest increase in segments of all URM participants. In addition, when we explored differences in racial/ethnic subgroups, some of which have a small sample for the analysis, we noticed that Latinx students had a different pattern of pre/post change in comparison with other URM groups. Although these differences were not statistically significant, Latinx students, on

\footnotetext{
${ }^{4} \mathrm{URM}=$ stands in this paper for underrepresented racial and/or ethnic minorities, such as African American, Latino/a/x, Native American, Pacific Islander and those who identify as multi-race/ethnicity.
} 
average, saw a slight decrease in most measures whereas almost all other groups slightly improved in most measures.

When we looked at students by major, engineering students' responses to the post-test showed increased mention of context segments compared to their pre-test responses, in contrast to participants pursuing a degree in computer science (CS). Students in computer science exhibited the largest increase in detail segments, which was statistically significant $(p<0.05)$. For CS students, the number of detail segments increased, on average, about one and a half segments. There is evidence to suggest that engineering students showed statistically significant increase in the absolute differences in context segments $(\mathrm{p}<0.01)$ with an increase on average of approximately one point.

The sample included a small number of students from humanities and social science fields who enrolled in the course $(n=6)$ and/or students who were undeclared $(n=4)$. The patterns of pre/post change were different among this small group as compared to other students.

Participants in humanities and/or social sciences tended to score higher in the pretest and showed the lowest increment in their metrics at the end of the course. These different responses merit deeper investigation with larger samples of these students.

We analyzed the data by aggregated cohort in order to maximize statistical power for comparisons (See Table 3 for groups). The research team examined "undergraduates" in their first, second, or third years compared with "advanced undergraduates" (seniors) and "graduate students". The data suggest no statistically significant changes in the absolute differences in segments neither for any of the cohort groups. However, in supplemental analysis not presented here, there is evidence that suggests a strong performance of advanced undergraduates (senior year) as compared with other undergraduate students. Seniors improved significantly in their number of segments, especially context segments $(p<0.05)$.

Additionally, graduate students exhibit a different pre/post differences pattern than do other groups. For instance, graduates gave, on average, less segments in the post, whereas all other groups improved. It is important to recognize that there were only five graduate-level students enrolled in the ENGR-Diversity course. 
Table 3. Absolute changes pretest and posttest differences compared within group

[Change in number of] Segments

\begin{tabular}{|c|c|c|c|c|c|c|}
\hline Groups & Subgroups & Average & Detail & Context & CSA & $\mathbf{N}$ \\
\hline \multirow{2}{*}{ نั } & Self-identified female & 0.42 & -0.34 & 0.76 & $1.61^{* * *}$ & 38 \\
\hline & Self-identified male & 0.73 & 0.42 & 0.30 & $2.15^{* * *}$ & 33 \\
\hline \multirow{2}{*}{ 递莞 } & Non URM & 0.52 & 0.15 & 0.38 & $1.69^{* * *}$ & 48 \\
\hline & URM & 1.27 & -0.76 & $2.03 *$ & $2.23^{* * *}$ & 24 \\
\hline \multirow{3}{*}{ 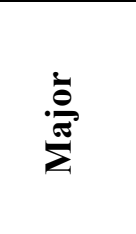 } & Engineering & 0.63 & -0.50 & $1.13^{* *}$ & $1.90^{* * *}$ & 40 \\
\hline & Humanities / Social Sciences & -3.17 & -1.50 & -1.67 & 0.17 & 6 \\
\hline & Computer Science & 1.29 & $1.29 *$ & 0.00 & $2.25^{* * *}$ & 24 \\
\hline \multirow{3}{*}{ 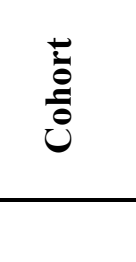 } & Undergraduates & 1.20 & 0.30 & 0.90 & $1.61 * * *$ & 28 \\
\hline & $\begin{array}{l}\text { Advanced Undergraduates / } \\
\text { Graduates }\end{array}$ & 0.60 & -0.07 & 0.67 & $1.98 * * *$ & 43 \\
\hline & Average & 0.72 & 0.04 & 0.68 & $1.88 * * *$ & 74 \\
\hline
\end{tabular}

${ }^{*}$ p-value $<.05,{ }^{* *}$ p-value $<.01,{ }^{* * *}$-value $<.001$, for difference within group pre/posttest change generated with a paired two sided $t$-test. Statistical analysis was only performed for larger groups. Numbers may not add up because students may have not identified with specific groups and/or have not declared a major.

\section{Contextual Social Awareness (CSA) analysis}

The new contextual social awareness framework makes it possible to investigate change in social understandings of a problem by looking at the distribution of students' responses (see Figure $3 \& 4$ ) and analyzing the additional CSA data. The results provide valuable insights into the changes in the nature of students' responses after the course.

Every segment that a student offered was given a 0 or 1 , depending on whether it contains social aspects. Students could have a pre- and post- CSA score equal to the number of segments they offered. So a number of two in terms of change in CSA as shown in the CSA column in Table 3 means that those students offered, on average, two more CSA segments in the posttest than they did in the pretest. Participants showed a significant gain in the number of socially aware segments across different groups in their pre-post responses. There is evidence to suggest that students of most subgroups, except for those majoring in the humanities or social sciences, showed a statistically significant improvement in the absolute differences in their CSA segments between pretest and posttest (see Table 3). Although we did not find statistically significant differences between the pretest and the posttest results for CSA for students in the 
humanities and social sciences, the lack of measurable difference may have been a consequence of the small sample size $(n=6)$.

The subgroups with the greatest improvements, on average, in the number of Contextual Social Awareness (CSA) segments are students who self-identified as male, URM, or were majoring in computer science. All of these subgroups showed an increase in the number of CSA segments, on average, above two points in the CSA segments. Additionally, Advanced Undergraduates/Graduates also improved by almost two CSA segments. The five graduates in this group increased by 2.2 points in CSA segments, while the 38 seniors in the group by 1.95 CSA segments. The difference between the tests results for the outcome measure, which was about 20-percentage point increase, may not likely have occurred by chance $(\mathrm{t}=6.819 ; \mathrm{df}=73$; $\mathrm{p}<0.0001$, see Table 4). Table 4 below shows the distribution of CSA segments by assessment type.

Table 4. Student's t-test results for pre/post number of CSA segments

\begin{tabular}{lcccc}
\hline & Average & $\begin{array}{c}\text { Standard } \\
\text { Deviation }\end{array}$ & t-value & Significance \\
\hline Pretest & 1.32 & 1.52 & 6.819 with 73 d.f. & \\
\hline Posttest & 3.01 & 2.40 & & $<.0001$ \\
\hline
\end{tabular}

Notice that after the course, the distribution of students' Contextual Social Awareness (CSA) is greater when compared to the pretest results. As shown in Table 4, there was a statistically significant difference between the mean of socially aware segments after the course compared to before the course. Participants scored significantly higher, on average, in the degree of social consideration after their participation in the class (mean $=3.01$ ) when compared to their pretest results $($ mean $=1.32)$. Students increased the number of Contextual Social Awareness (CSA) segments after the course at a statistical level of $\mathrm{p}<0.001$. When pondering the design problem scoping in week 9 of the course, students included more factors in their responses related to human diversity and broader social issues.

The data suggest there were important differences within groups in their consideration of contextual social awareness (CSA). To further explore these differences, a two-way analysis of variance was conducted on the influence of various dependent variables, such as gender, race/ethnicity, major, and cohort. We conducted supplemental analyses, not included in this manuscript, to investigate the differences in CSA segments in all groups. We especially focused our analysis on gender, race \& ethnicity and major as the data suggest these groups exhibited the greatest increases in the outcome variable in the paired t-test analysis (see Table 3), performing analysis of the interaction between the independent variables. We found that constructs considering gender and major taken together yielded a statistically significant difference, while all the other interactions were not statistically significant. The variable gender included two levels (Self-identified Female and Male) and major consisted of four levels (Engineering, Computer Science, Humanities and Undeclared). The Analysis of Variance (ANOVA) met the assumptions of normality and homogeneity of variance by generating and analyzing 
visualizations of the data. As shown in Table 5, none of the effects were statistically significant except for the interaction effect. A post hoc Tukey's Honestly Significant Difference (HSD) test suggests that undergraduate female students enrolled in the humanities and social sciences (mean $=-1.50, \mathrm{n}=4)$ and male students in computer science $($ mean $=2.76, \mathrm{n}=13)$ differed significantly in their change in CSA segments $(p<.05)$. The data show no evidence for statistically significant differences in any other group when controlling for gender and major. Although the only statistically different groups were self-identified females in humanities/social sciences when compared with self-identified male in computer science, we need to understand the limitations of the small sample size for these groups.

Table 5. ANOVA results

\begin{tabular}{|c|c|c|c|c|c|c|}
\hline Variables & $F$ ratio & Degrees of Freedom & Error & $\mathbf{p}$ & Mean change CSA & Standard deviation \\
\hline Gender & 1.36 & 1 & 66 & $>.05$ & & \\
\hline $\begin{array}{l}\text { Self-identified } \\
\text { Women }\end{array}$ & & & & & 1.59 & 2.39 \\
\hline $\begin{array}{l}\text { Self-identified } \\
\text { Men }\end{array}$ & & & & & 2.20 & 2.33 \\
\hline Major & 1.24 & 3 & 66 & $>.05$ & & \\
\hline Engineering & & & & & 1.90 & 2.02 \\
\hline Computer Science & & & & & 2.25 & 2.40 \\
\hline Humanities & & & & & .17 & 3.87 \\
\hline Undeclared & & & & & 2.00 & 2.70 \\
\hline Gender*Major & 3.33 & 3 & 66 & $<.05$ & & \\
\hline
\end{tabular}

\section{Discussion}

The research presented here expands the Midwest Flooding Problem (MWF) framework by adding new dimensions to explicitly capture a new element for that framework: Contextual Social Awareness (CSA). The results suggest an increase in social dimensions in students' responses, which prompted a revision to the MWF coding.

In answering the first research question on the extent in which students consider social issues when solving design problems, we found that participants increase their consideration to social and broader issues in their design. The data suggest that the variables gender and major may significantly influence students' outcomes in terms of their change of CSA segments between pretest and posttest. The second research question asks about any changes in students' social awareness after they participate in a course on culture, diversity, and equity in engineering communities? The results suggest that students considered in their responses local users, socioeconomic status, culture, power, access, diversity, minorities and separation of 
communities. Students' considerations of broader social factors have potentially not occurred by chance.

The statistically significant increase of contextual social awareness shows that students can change their design consideration within a short time frame of roughly two months. Students increase the number of design factors considered related to human diversity and broader social issues when pondering the design problem scoping. The only slight increase was the CSA segments of students from the humanities. The small sample for the humanities subgroup or their higher pretest results as compared with engineering and computer science students are plausible explanations for their minor improvement, which was not statistically significant, in the CSA segments. In other words, students in the humanities potentially come more into contact with contextual social issues, as compared to students in the technical fields, and might therefore not exhibit as much change in CSA as a result of courses such as ENGR-Diversity as do engineering and computer science students.

The data also suggest a statistically significant difference between self-identified female students in the humanities compared to self-identified male students in computer science. Thus, the type of program one is enrolled in interaction with gender potentially influences one's ability to consider broader factors in engineering design. However, further research is needed to better understand this interaction because of the small sample size of both of these subgroups.

For the changes in Contextual Social Awareness segments, we found no significant differences in the increase between groups. We believe that this finding is an important result, showing that, regardless of demographic background, factors such as age, field of studies, sex, ethnicity, people all change in a similar way and can learn to incorporate contextual social awareness into design projects within a short period of two months. No matter who is currently working on design projects, we all can learn to be more aware of social factors. Students of all backgrounds can learn to incorporate social dimensions right from the start of their career.

Although we cannot make causal claims about the effects of the course, the findings suggest that the course may have helped students recognize the importance of broader issues and social context in solving engineering design problems. We draw this inference from multiple factors, especially considering students' answers in the posttest, the increment in context segments for key groups, differences between groups decreased and the significant increase of social awareness scores for most groups. These outcomes shed additional light and are in line with previous evaluation of the impact of such courses [28].

\section{Limitations}

Due to a missing control group, the study design does not allow for a causal relation to be established between the test results and the impact of the course experience. A study on the effect of the course could be performed with a control group and additional tests to check for progress throughout the course. Another study design could be to test students after different course elements or expose them to only a single course element to identify which parts of the course had the biggest influence on the design task. 
Though there were multiple indications that the course could have an impact on Contextual Social Awareness, this hypothesis needs to be further investigated in the future to make sure it is not due to other factors we did not consider. Additionally, some of the subgroups have a relatively low sample size, such as graduate students $(n=5)$ and groups with intersectional identities such as Latinas $(n=2)$. A sample with larger numbers of graduate and minority students could test the preliminary findings of this paper. Data collecting and analysis from a small sample size, while limited, still did offer some very useful information that warrants further investigation.

We need to interrogate the results for long term effects of the potential benefits of the course experience. The findings might only be valid for a short time and within the context of the course. The classroom environment encouraged students to think and openly talk about highly sensitive issues in a safe space. It is not clear how a different environment would impact the results. For instance, how would students respond in a work setting with colleagues of different ages or power relations. The different settings raise the question of how learning about diversity in engineering may affect engineers already in the workforce. If the findings did transfer to workplaces, we might find that it would be beneficial for employers to engage employees in learning about ENGR-Diversity course topics, since within a short time frame of ten weeks it seems possible to change how people think about design. Testing the effects of such learnings in the workplace could extend the findings to value within a work setting.

Moreover, students might have answered what they think the teaching team would expect, not what they truly would consider. This external expectation, even though at no time consciously addressed by the research or teaching team, might have had an impact on the students' responses as they tried to fulfill what they perceived to be the expectations. These environmental issues could be resolved by repeating the test in a workplace setting as well as giving practitioners a course experience, such as ENGR-Diversity.

Additionally, our sample was more diverse than the average engineering population. It was more diverse in terms of gender, ethnicity and race, and different disciplines. This might have had an impact on the learning of topics around diversity, as students could contribute and share their own personal experiences. It might also have had an impact on the willingness to learn or the general awareness for the problem and its necessity.

Another general question is if teamwork would generally change or impact the results. As design work usually takes place in a team, team dynamics are important and might lead to different results compared to single student responses. The binary CSA is not tested extensively by different researchers and samples and therefore has a limited reliability. However, the CSA aims to spark a broader discussion between design researchers that emphasizes the potential of more carefully measuring social factors as an invaluable source of information about the students. 


\section{Conclusion}

Future research could focus on investigating the link between changes in students' Contextual Social Awareness (CSA) and the pedagogical and instructional elements of the course. More specifically, which aspects of the course contributed to the changes in students' responses. Additionally, the binary measure of contextual social awareness is a first step, which could be extended by other factors to create a more sophisticated point scale representing more accurately the nuances of the students' answers. Furthermore, the CSA measure focuses primarily on human factors. An extension of the CSA measure that considers aspects of the natural environment might add important contributions to this framework.

Social aspects of engineering are important and will become even more so due to demographic shifts. At the same time, two important mismatches prevail in the engineering field. First, an engineering workforce with a demographic that does not reflect the diversity of the overall population. Second, discrepancies between the skills engineering students develop in school, which are primarily technical, and those needed in practice. To improve engineering courses, design researchers need to develop pedagogical strategies for social awareness and tools that can effectively measure their effectiveness.

After participating in the ENGR-Diversity course, most students increased their number of contextual segments and significantly increased the number of CSA segments in the posttest. Furthermore, students developed a greater understanding of social considerations as exemplified by the quotes from students like Jose and Yulin. The results reported here suggest that students changed their design considerations about social issues within a short time frame, which signal a potential positive influence of the ENGR-Diversity course on students' design considerations. We can expand current frameworks to investigate and measure design considerations to account for broader social aspects in engineering. The proposed binary Contextual Social Awareness (CSA) extends the scope and depth of the classic MWF framework. The CSA adds valuable insights for researchers and educators into the nature of responses.

The most interesting insights of this work resulted from centering participants' voices by investigating closely the original quotes from students. The key to creating innovation, also in innovative research itself, is a deep understanding of the user [29]. This study is a further example that it is always worthwhile to invest time to deeply understand the participants and to take a close look at the original dataset.

\section{Acknowledgements}

The author team wishes to acknowledge the students in the course described in the paper. These students are our inspiration for striving to bring culture and diversity more centrally into engineering curriculum. Financial support by the Stanford Vice Provost of Undergraduate Education (through a Faculty College Grant), the School of Engineering Dean's Office (and especially Prof. Tom Kenny), and a General Motors gift to support diversity and inclusion programming are gratefully recognized. 


\section{References}

[1] L. S. Dankert, S. K. Gilmartin, C. B. Muller, C. Dungs, S. D. Sheppard and C. LeichtScholten, "Expanding engineering limits: a concept for socially responsible education of engineers." The International journal of engineering education, vol. 35, no. 2, pp. 658673, 2019.

[2] S. D. Sheppard, K. Macatangay, A. Colby and W.M. Sullivan, Educating Engineers: Designing for the Future of the Field. San Francisco, CA: Jossey-Bass, 2009.

[3] H. J. Passow and C. H. Passow, "What competencies should undergraduate engineering programs emphasize? A systematic review.” Journal of Engineering Education, vol. 106, no. 3, pp. 475-526, 2017.

[4] E. A. Cech, "The (mis)framing of social justice: why meritocracy and depoliticization hinder engineers' ability to think about social injustices." in Engineering Education for Social Justice: Critical Explorations and Opportunities, J.C. Lucena, Ed. New York: Springer, 2013, pp. 67-84.

[5] J. Trevelyan, "Reconstructing engineering from practice." Engineering Studies, vol. 2, no. 3, pp. 175-195, 2010.

[6] T. Brown, "Design Thinking." Harvard Business Review, vol. 86, no. 6, pp. 84-92, 2008.

[7] B. Johansen and J. Euchner, "Navigating the VUCA world." Research-Technology Management, vol. 56, no. 1, pp. 10-15, 2013.

[8] P.D. Galloway, "The 21st-Century Engineer. A Proposal for Engineering Education Reform.” Civil Engineering Magazine Archive, vol. 77, pp. 46-104, 2008.

[9] CBInsights (ed.), "2020 Tech Trends", CBInsights Research Reports, pp. 45-48, January 22, 2020. [Online]. Available: CBInsights, https://www.cbinsights.com/research/report/top-tech-trends-2020/ [Accessed January 26, 2020]

[10] C. Corbett and C. Hill, "Solving the equation: The variables for women's success in engineering and computing." The American Association of University Women, California, Research Report, March, 2015.

[11] A. Burke, "Science and Engineering Labor Force." in National Science Board, National Science Foundation. 2019. Science and Engineering Indicators 2020. NSB-2019-8. Alexandria, VA, 2019, pp. 6-7. [Online]. Available at https://ncses.nsf.gov/pubs/nsb20198/. [Accessed: April 28, 2020].

[12] WM. A. Wulf, "The Importance of Diversity in Engineering - National Academy of Engineering" in Diversity in Engineering: Managing the Workforce of the Future, C. R. Arenberg, Ed. Washington, DC: The National Academies Press, 2002, pp. 8-14.

[13] K. W. Phillips, "How Diversity Makes Us Smarter.” Scientific American. September 16, 2014. [Online]. Available: Scientific American, http://www.scientificamerican.com/article/how-diversity-makes-us-smarter. [Accessed: April 29, 2020].

[14] D. Riley, A. Slaton and A. Pawley, "Social Justice and Inclusion." in Cambridge Handbook of Engineering Education Research, A. Johri and B. Olds, Eds. Cambridge: Cambridge University Press, 2014, pp. 335-356. [E-book] Available: Cambridge.org

[15] S. Tolbert, A. Schindel, and A. J. Rodriguez, "Relevance and relational responsibility in justice oriented science education research." Science Education, vol. 102, no. 4, pp. 796819, 2018. 
[16] E. Suntano "Turning Diversity into Competitive Advantage: A Case Study of Managing Diversity in the United States of America." Jurnal Manajemen Dan Kewirausahaan, vol. 11, no. 2, pp. 154-160, 2009.

[17] W. Vincenti, What Engineers Know and How They Know It. Baltimore: Johns Hopkins Press, 1990.

[18] E. A. Cech and T. J. Waidzunas, "Navigating the heteronormativity of engineering: The experiences of lesbian, gay, and bisexual students." Engineering Studies, vol. 3, no. 1, pp. $1-24,2011$

[19] J.C. Garibay, "STEM students' social agency and views on working for social change: Are STEM disciplines developing socially and civically responsible students?" Journal of Research in Science Teaching. vol. 52, no. 5, pp. 610-632, 2015.

[20] N. Dukhan, M.R. Schumack and J.J. Daniels, "Service Learning as Pedagogy for Promoting Social Awareness of Mechanical Engineering Students." International journal of Mechanical Engineering Education, vol. 37, no. 1, pp. 78-86, 2012.

[21] C.H. Joslyn and M.M. Hynes, "Measuring Changes in Self-awareness and Socialawareness of Engineering Students' Engaging in Human-Centered Design." in Educational Research and Methods Proc. 2016 ASEE Annual Conf. \& Expo., New Orleans, LA, June 26-29, 2016.

[22] E.A. Cech “Culture of Disengagement in Engineering Education?” Science, Technology \& Human Values, vol. 39, no. 1, pp. 42-72, 2014.

[23] C. J. Atman, K. Yasuhara, R. S. Adams, T. J. Barker, J. Turns and E. Rhone, "Breadth in problem scoping: A comparison of freshman and senior engineering students." International Journal of Engineering Education, vol. 24, no. 2, pp. 234-245, 2008.

[24] C. J Atman, M. E. Cardella, J. Turns, and R. Adams, "Comparing freshman and senior engineering design processes: an in-depth follow-up study", Design Studies, vol. 26, no. 4, pp. 325-357, 2005.

[25] M. E. Cardella, C. J. Atman, J. Turns and R. Adams, "Students with Differing Design Processes as Freshmen: Case Studies on Change." In Proc. Clive L. Dym Mudd Design Workshops VI: Design and Engineering Education in a Flat World, Harvey Mudd College, Clermont, CA, June 2007.

[26] C. A. Mullins, C. J. Atman and L. J. Shuman, "Freshman engineers' performance when solving design problems." IEEE Transactions on Education, vol. 42, no. 4, pp. 281-287, 1999.

[27] G. Pérez, S. K. Gilmartin, C. B. Muller, P. M. Danner and S. D. Sheppard, "Design Problems in Context: A Longitudinal Examination of Students' Design Considerations in a Course about Engineering Culture, Diversity, and Equity." In Proc. Clive L. Dym Mudd Design Workshops XI: Design Education \& Practice "How Process Matters, Harvey Mudd College, Clermont, CA, May 30 - June 01, 2019.

[28] C. N. Onyeador, S. K. Gilmartin, G. Trujillo, C. M. Muller and S. D. Sheppard, "Adding Diversity and Culture to the Engineer's Toolkit: Evaluating a Unique Course Option for Engineering Students." Creating Equity Through Structure and Pedagogy Proc. 2018 ASEE Annual Conf. \& Expo., Salt Lake City, UT, June 24-27, 2018.

[29] T. Kelley and D. Kelley, "Reclaim Your Creative Confidence." Harvard Business Review, vol. 90, no. 12, pp. 115-118, 2012. 